\title{
Análise dos esquemas de insulinoterapia prescritos aos pacientes com hiperglicemia de um hospital universitário de uma cidade do sul de Minas Gerais
}

\author{
Analysis of insulin therapy schemes prescribed to patients with hyperglycemia in a \\ university hospital in a southern city of Minas Gerais
}

Análisis de los regímenes de terapia con insulina prescritos a pacientes conhiperglucemia en un hospital universitario en una ciudad del sur de Minas Gerais

Brenda Gersanti Borba ${ }^{1 *}$, Amanda Nascimento Areias Forster ${ }^{1}$, Gabrielly Pelegrini Domingues ${ }^{1}$, Mayra Lima de Carvalho ${ }^{1}$, Roberta Bessa Veloso Silva, Gérsika Bitencourt Santos.

\section{RESUMO}

Objetivo: Avaliar os esquemas de insulinoterapia Basal-bolus (B-B) e Sliding Scale Insulin (SSI) utilizados em pacientes internados em um hospital universitário do sul de Minas Gerais. Métodos: Estudo transversal exploratório, realizado com 50 pacientes. Foram analisados prontuários de pacientes internados no setor de clínica médica de um hospital universitário do sul de Minas Gerais, que usaram insulinoterapia para o controle da hiperglicemia. Os pacientes foram classificados quanto aos dados sociodemográficos, tipo de insulina utilizada e presença de hipoglicemia. Foi assinado o Termo de Consentimento Livre e Esclarecido e os dados foram coletados de modo descritivo, no período de seis meses (fevereiro de 2017 a agosto de 2017). O estudo foi aprovado por Comitê de Ética em Pesquisa. Resultados: Durante a internação, 32 pacientes (64\%) utilizaram o esquema SlidingScale, 2 pacientes (4\%) usaram o esquema de insulina Basal Bolus e 16 pacientes (32\%) fizeram uso de ambos, SSI e BB. A menor média glicêmica encontrada foi em pacientes do grupo B-B (156,04 mg/dl) e, a maior, do grupo SSI (210,18 mg/dl). Conclusão: A grande maioria dos pacientes hospitalizados, que apresentam hiperglicemia, receberam o esquema SSI. É necessário realizar novos estudos comparativos entre os esquemas insulinoterápicos, avaliando o mais eficaz.

Palavras-chave: Diabetes mellitus, Glicemia, Insulinoterapia.

\begin{abstract}
Objective: To analyze the basal bolus (B-B) and sliding-scale insulin (SSI) insulin therapy schemes used in patients admitted to a university hospital in southern Minas Gerais. Methods: Cross-sectional exploratory study, carried out with 50 patients. Medical records of patients admitted to the medical clinic sector of university hospital in southern Minas Gerais, who used insulin therapy to control hyperglycemia were analyzed. Patients were classified according to sociodemographic data, type of insulin used and presence of hypoglycemia. The Informed Consent Form was signed and the data were collected in a descriptive manner, with no period of six months (February 2017 to August 2017). The study was approved by the Research Ethics Committee. Results: During hospitalization, 32 patients (64\%) used the Sliding Scale scheme, 2 patients (4\%) used the basal Bolus insulin scheme and 16 patients (32\%) used both SSI and BB. The lowest glycemic average found was in patients in the B-B group (156.04 mg / dl) and the highest in the SSI group $(210.18 \mathrm{mg} / \mathrm{dl})$. Conclusion: The vast majority of hospitalized patients, who have hyperglycemia, received the SSI regimen. It is necessary to conduct new comparative studies between insulin therapy regimens, evaluating the most effective.
\end{abstract}

Keywords: Diabetes mellitus, Glycemia, Insulin therapy.

${ }^{1}$ Universidade José do Rosário Velano (UNIFENAS), Alfenas - MG.

*E-mail: brendagersanti@gmail.com

SUBMETIDO EM: 7/2020

I ACEITO EM: $8 / 2020$

PUBLICADO EM: 10/2020

REAS / EJCH | Vol.12(10) | e4564 | DOI: https://doi.org/10.25248/reas.e4564.2020 Página 1 de 9 


\section{RESUMEN}

Objetivo: Analizarlos esquemas de terapia de insulina con bolo basal (B-B) e insulina de escala móvil (SSI) utilizados en pacientes ingresados en un hospital universitario en el sur de Minas Gerais. Métodos: Estudio exploratorio transversal, realizado con 50 pacientes. Se analizaron los registros médicos de los pacientes ingresados en el sector de la clínica médica del hospital universitario en el sur de Minas Gerais, que usaron terapia con insulina para controlar la hiperglucemia. Los pacientes se clasificaron según los datos sociodemográficos, el tipo de insulina utilizada y la presencia de hipoglucemia. Se firmó el formulario de consentimiento informado y los datos se recopilaron de manera descriptiva, sin un período de seis meses (febrero de 2017 a agosto de 2017). El estudio fue aprobado por el Comité de Ética en Investigación. Resultados: Durante la hospitalización, 32 pacientes (64\%) usaron el esquema de Escala móvil, 2 pacientes (4\%) usaron el esquema de insulina basal en bolo y 16 pacientes (32\%) usaron SSI y BB. EI promedio glucémico más bajo encontrado fue en pacientes en el grupo B-B $(156.04 \mathrm{mg} / \mathrm{dl})$ y el más alto en el grupo SSI $(210.18 \mathrm{mg} / \mathrm{dl})$. Conclusión: La gran mayoría de los pacientes hospitalizados, que tienen hiperglucemia, recibieron el esquema SSI. Es necesario realizar nuevos estudios comparativos entre los regímenes de terapia con insulina, evaluando los más efectivos.

Palabras clave: Diabetes mellitus, Glucemia, Terapia con insulina.

\section{INTRODUÇÃO}

A glicemia é regulada, em suma, pela insulina e pelo glucagon, hormônios sintetizados nas ilhotas pancreáticas de Langerhans (GELONEZE B, et al., 2006). Em pacientes portadores de Diabetes mellitus (DM), um dos distúrbios mais prevalentes na saúde pública mundial, há a presença de hiperglicemia, decorrente de defeitos na ação hormonal da insulina, na sua secreção ou de ambos, como referido nas Diretrizes da Sociedade Brasileira de Diabetes (2015-2016).

A hiperglicemia nos doentes críticos e hospitalizados é um fenômeno recorrente, mesmo naqueles sem diabetes conhecida. Além de hiperglicemia característica no DM, é comum haver hiperglicemia de estresse em pacientes internados possuidores de enfermidades graves, como sepse grave e choque séptico. Essa hiperglicemia de estresse acarreta diminuição da função imunológica e aumento do estresse oxidativo e dos fatores inflamatórios, desencadeando em posteriores complicações (VIANA MV, et al., 2014; LISBOA, HRK, et al., 2000). A resposta ao estresse é devido a uma interação complexa entre hormônios contrareguladoras, citocinas e alterações na sensibilidade à insulina (GELONEZE B, et al., 2006).

A hiperglicemia manifesta-se por sintomas como poliúria, polidipsia, perda de peso, polifagia e visão turva ou por complicações agudas que podem levar a risco de vida, como a cetoacidose diabética e a síndrome hiperosmolar hiperglicêmica não cetótica. Estudos demonstram que, um melhor controle glicêmico, é eficaz contra o aparecimento de complicações relacionadas ao DM (GÓES, APP et al., 2007)

Sabe-se que a DM é uma afecção muito prevalente, ela representa $5,2 \%$ das causas de mortes no Brasil e, assim como outras doenças crônicas, atinge especialmente grupos populacionais vulneráveis como idosos, pessoas de baixa renda e baixa escolaridade. Dessa forma, é notável que ela seja um tema de interesse para a saúde pública. (FREITAS LRS; GARCIA LP, 2012)

Alguns estudos indicam que a melhora do controle glicêmico leva a ocorrência de menores taxas de complicações hospitalares e mortalidade. (GALINDO $R$ e UMPIERREZ GE, 2018). Dessa forma, a intervenção para minimizar os níveis glicêmicos consiste em mudanças nos hábitos de vida, como a prática de atividades físicas ou orientações dietéticas, e também no uso de medicações, isoladas ou em associações, como os antidiabéticos orais ou injetáveis como a insulina (ARAÚJO LMB, et al., 2000).

Em relação à insulinoterapia, existem dois diferentes esquemas a serem utilizados: Basal Bolus (B-B) e SlidingScale (SSI). O esquema de insulinoterapia Basal-Bolus, trata-se de um tratamento feito antes da ocorrência da hiperglicemia, ou seja, visando prevení-la. Nesse, há o esquema Basal, com o uso de insulina glargina, detemir e NPH, associado à insulina em bolus, representada por regular, aspart, lispro e glulisina, que previne a hiperglicemia de jejum e controla a glicemia após as refeições (UMPIERREZ GE, et al., 2007). 
O esquema SlidingScale se refere à aplicação da insulina apenas quando o paciente possuir uma hiperglicemia. Deste modo, esse método é geralmente composto de insulina regular, sem quaisquer insulinas intermediárias ou de longa duração. Além disso, trata a hiperglicemia após ela já ter ocorrido, em vez de prevenir sua ocorrência (CLEMENT S, et al., 2004).

O estudo em pauta visa identificar os pacientes hiperglicêmicos de um hospital universitário do sul de Minas Gerais, objetivando, assim, comparar os esquemas de insulinoterapia prescritos e classificá-los segundo variáveis pré-estabelecidas.

É importante o tratamento insulinoterápico devido aos riscos causados pela hiperglicemia. Atualmente, existem vários esquemas disponíveis para o uso hospitalar e por isso é importante buscar aquele que é mais benéfico para o paciente, visando diminuir a incidência de hiperglicemia e, também, de hipoglicemia.

\section{MÉTODOS}

Foi realizado um estudo transversal, através de uma pesquisa exploratória que comparou diversos pacientes que fazem uso dos esquemas de administração de insulina basal-bolus e sliding-scale.

Pacientes internados no setor de Clínica Médica de um hospital universitário do sul de Minas Gerais, com prescrição de controle de glicemia capilar através de insulina, foram selecionados. Os pacientes assinaram o Termo de Consentimento Livre e Esclarecido (TCLE) ao serem admitidos no hospital. Os dados foram coletados de modo descritivo, através do prontuário médico no período de seis meses (fevereiro de 2017 a agosto de 2017).

Dessa forma, cinquenta (50) pacientes foram selecionados para o estudo. Não houve restrição quanto ao gênero, aos medicamentos utilizados, aos diagnósticos prévios, à idade ou ao motivo da internação. Foram excluídos da amostra aqueles que possuíam dados insuficientes no prontuário médico, aqueles que permaneceram internados menos de dois dias e pacientes que foram internados em outro setor do hospital.

O tipo de insulina basal-bolus administrado aos pacientes foi a NPH e, dentro do esquema slidingscale, insulina regular.

Os dados coletados nos prontuários foram registrados em um instrumento elaborado para esse estudo. Nesse instrumento havia os seguintes tópicos: sexo do paciente, idade, motivo da internação, diagnóstico prévio de DM, esquema de insulinoterapia utilizado, glicemia de jejum e presença de hipoglicemia.

Para a análise dos dados utilizou-se o teste de qui-quadrado, ao nível nominal de $5 \%$ de significância para verificar a existência de associação entre as variáveis analisadas. O teste exato de Fisher foi aplicado nos casos em que a frequência absoluta das células da tabela de contingência foi menor ou igual a 5 .

Os dados foram organizados e apresentados pelo gráfico de barras e de setores. A análise estatística foi realizada no software $R$ ( $R$ CORE TEAM, 2020). Para o cálculo amostral foi definido um nível de significância de $95 \%$ e uma margem de erro de 0,05 .

Este projeto foi realizado após aprovação no Comitê de Ética em Pesquisa da Universidade José do Rosário Vellano/UNIFENAS sob protocolo número 1.877.305., conforme a Resolução 466/12, Comissão Nacional de Saúde/Ministério da Saúde, de 13/06/13. Comitê de Ética em Pesquisa (CAE 62965316.9.0000.5143).

\section{RESULTADOS}

Dos 50 pacientes envolvidos na pesquisa, 24 (48\%) são do sexo feminino e 26 (52\%) pacientes do sexo masculino. Em relação à faixa etária, $6 \%$ dos pacientes tinham até 40 anos, $18 \%$ estavam entre 40 a 59 anos e $76 \%$ apresentavam idade superior a 60 anos (Gráfico 1). 
Gráfico 1 - Faixa etária dos pacientes.

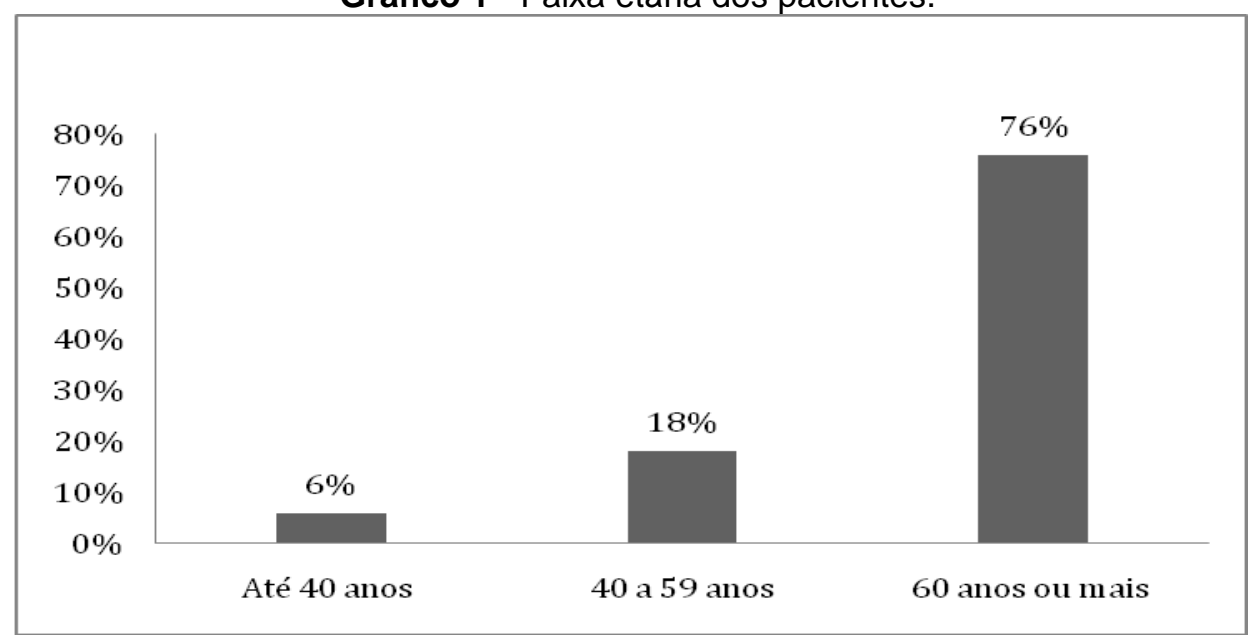

Fonte: Borba BG, et al., 2020.

Em relação ao motivo relacionado às internações dos pacientes abordados em relação ao sexo, destacase a pneumonia (13 pacientes). Outros motivos recorrentes foram AVC (7 pacientes) e outros problemas neurológicos (9 pacientes). As complicações diabéticas, por sua vez, levaram a $3(4,6 \%)$ internações. A análise da relação entre estas duas variáveis (motivo da internação $x$ sexo), não se mostrou significativa, ao nível de $5 \%$ de significância, pelo teste exato de Fisher ( $p=0,7251$ ) (Gráfico 2).

Quando questionados sobre o diagnóstico prévio de diabetes, 18 (75\%) pacientes do sexo feminino e 19 (73\%) pacientes do sexo masculino tinham conhecimento da doença antes da internação diagnostico prévio diabetes, apenas 1 paciente do sexo masculino não informou sobre o diagnóstico prévio (Gráfico 3).

Durante a internação, 32 pacientes (64\%) utilizaram o esquema SlidingScale, 2 pacientes (4\%) usaram o esquema de insulina Basal Bolus e 16 pacientes (32\%) fizeram uso de ambos, SSI e BB, ou seja, usaram tanto a insulina do tipo regular (insulina de ação rápida) quanto a do tipo NPH (insulina de ação intermediária).

No presente estudo, os pacientes que utilizavam o esquema B-B tiveram a média glicêmica de 156,04 $\mathrm{mg} / \mathrm{dL}$. Os pacientes que receberam o esquema de insulina SSI apresentaram uma média glicêmica de $210,18 \mathrm{mg} / \mathrm{dl}$, enquanto os que foram tratados com esquema duplo (B-B+ SSI) a média foi 206,6052 mg/dl.

Gráfico 2 - Frequências absolutas dos motivos de internação de acordo com o sexo.

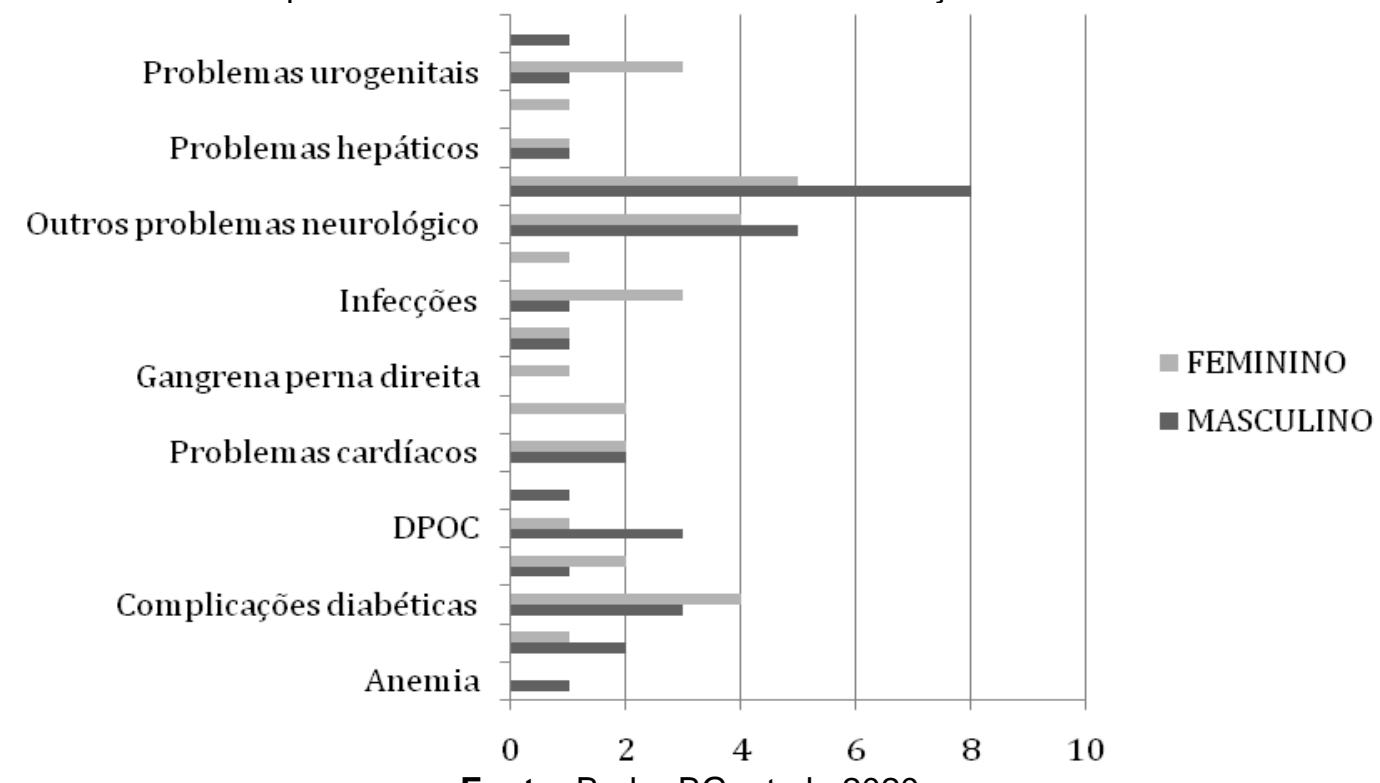

Fonte: Borba BG, et al., 2020. 
Gráfico 3 - Diagnóstico prévio de Diabetes mellitus.

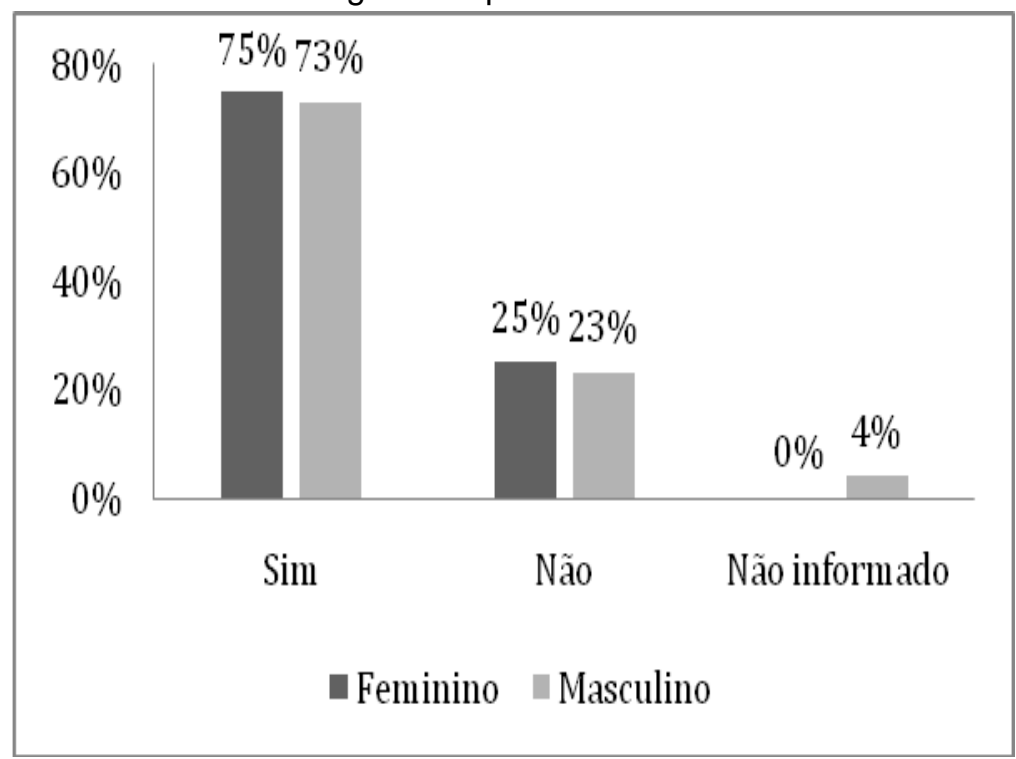

Fonte: Borba BG, et al., 2020.

De acordo com o Gráfico 4, verifica-se que, dos 26 (52\%) que receberam a dieta hipoglicêmica, 2 (8\%), utilizam o esquema B-B; 11 (42\%), o SSI; e $13(50 \%)$, o B-B+SSI. Dos 24 (48\%) que não receberam a dieta, 0 (0\%), utilizaram B-B; 21 (88\%), o SSI e $3(12 \%)$, o B-B+SSI. Pode-se observar que a frequência do tipo SSI foi 2 vezes maior entre os que não receberam a dieta hipoglicêmica ao se comparar com os que receberam a dieta. Já a frequência de uso da combinação B-B+SSI foi 4 vezes maior naqueles que receberam a dieta do que entre os que não receberam a dieta (Gráfico 4).

Quanto aos episódios de hipoglicemia, considerada neste estudo como glicemia capilar $<70 \mathrm{mg} / \mathrm{dL}$, 8 (16\%) pacientes apresentaram pelo menos 1 episódio de hipoglicemia durante a internação. Sendo que 1 $(50 \%)$ desses pacientes pertencem ao grupo B-B, $2(6,25 \%)$ ao SSI. Já no grupo B-B+SSI, a hipoglicemia esteve presente em $5(31,2 \%)$ dos pacientes (Gráfico 5 ).

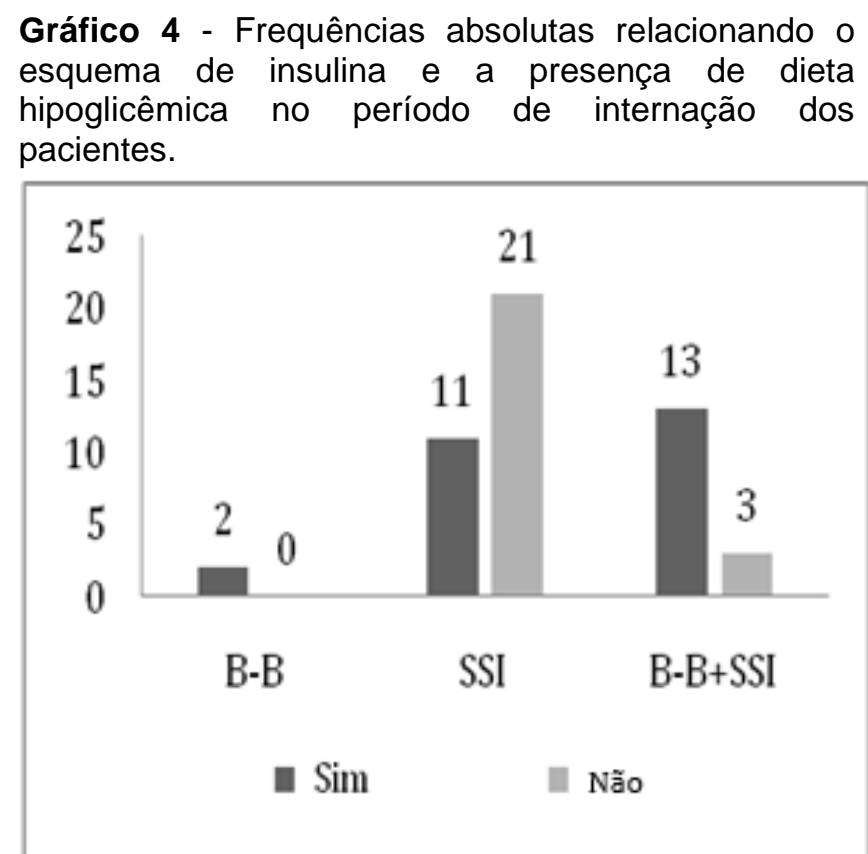

Fonte: Borba BG, et al., 2020. 
Gráfico 5 - Frequências absolutas relacionando o esquema de insulina e a presença de hipoglicemia dos pacientes.

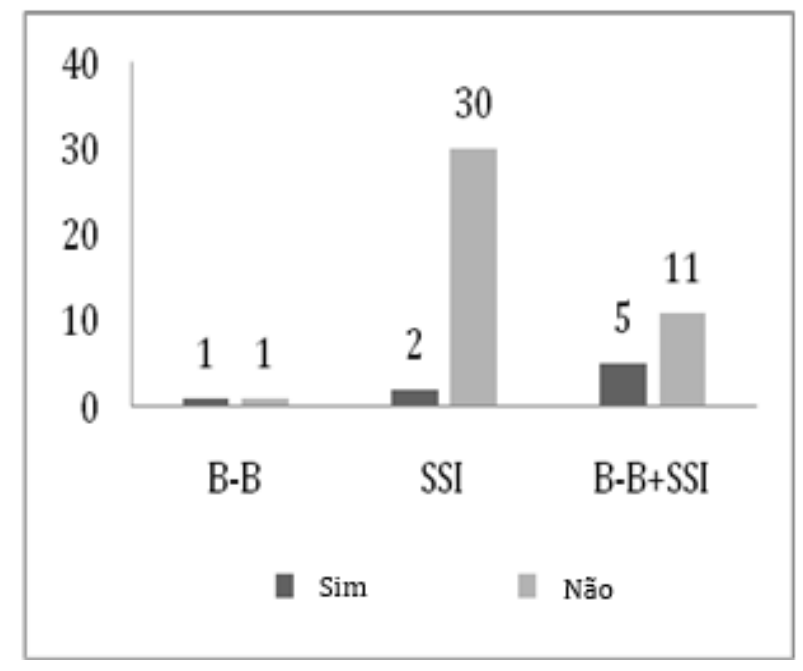

Fonte: Borba BG, et al., 2020.

\section{DISCUSSÃO}

Sabe-se que o Diabetes mellitus é uma doença caracterizada pelo comprometimento do metabolismo da glicose, resultando em hiperglicemia crônica (PETERMANN XB, et al., 2015). No presente estudo evidenciou-se uma amostra homogênea dos pacientes com hiperglicemia em relação ao sexo, sendo que, o número de mulheres da amostra foi ligeiramente menor do que o número de homens. Esses pacientes podem ter apresentado hiperglicemia de estresse ou hiperglicemia característica em pacientes portadores de Diabetes mellitus. Em outro estudo sobre a carga de Diabetes mellitus no Brasil, não se encontrou diferenças relevantes entre as incidências de diabetes entre homens e mulheres, sendo ligeiramente maior nos homens (COSTA AF, et al., 2017).

Os pacientes envolvidos neste estudo apresentaram hiperglicemia no ambiente hospitalar com necessidade de Insulinoterapia. No ambiente intra-hospitalar, é comum haver hiperglicemia de estresse em pacientes internados possuidores de enfermidades graves, assim como a hiperglicemia característica em pacientes portadores de Diabetes mellitus (VIANA MV, et al., 2014). Dessa forma, está bem estabelecida a associação entre hiperglicemia hospitalar em pacientes hospitalizados com e sem diabetes com mortalidade ininterrupta, morbidade, tempo de internação, infecções e número geral de complicações. Alguns estudos observacionais e randomizados controlados indicam que menores taxas de complicações hospitalares e mortalidade são resultados da melhora no controle glicêmico (GALINDO R e UMPIERREZ GE, 2018).

Concomitantemente, observou-se que a prevalência de pacientes com hiperglicemia em ambiente hospitalar foi maior em pacientes com mais de 60 anos. Sabe-se que a prevalência de pacientes com DM intrahospitalar aumenta com idade, gravidade da doença de base e a presença de comorbidades (ROSA R, et al., 2014).

Como citado anteriormente, os participantes desta pesquisa foram compostos de pacientes internados que fizeram uso de insulinoterapia para o controle da glicemia. Dessa forma, a própria presença de pneumonia pode ter promovido o aumento da glicemia capilar e, consequentemente levado ao uso de insulina nesses pacientes, elevando o número de internações relacionadas à pneumonia com uso de insulinoterapia. Alguns estudos indicam que a pneumonia pode exacerbar uma doença subjacente, como doença pulmonar obstrutiva, insuficiência cardíaca congestiva ou Diabetes mellitus (ARNOLD FW et al., 2003; MARRAS TK et al., 2000). Dessa forma, a prevalência de pneumonia nesse estudo poderia ser explicada devido à própria relação entre DM e pneumonia. 
As complicações diabéticas, por sua vez, levaram a $3(4,6 \%)$ internações. Essa porcentagem se assemelha com a encontrada por Alfradique, et al (2009) que mostrou que, das internações realizadas na rede hospitalar do SUS em 2006, houve mais de 120.000 internações só em relação ao DM, correspondendo a $4,3 \%$ de todas as internações por CSAP (Condições Sensíveis à Atenção Primária), a uma taxa de 6,5 por 10.000 habitantes. Sabe-se que ocorrência de internação por DM e suas complicações é um indicativo de falha na atenção aos pacientes portadores dessa doença crônica, que requer seguimento contínuo e prolongado, com ações de prevenção de complicações tais como o coma ou a cetoacidose diabética, além das complicações renais, oftálmicas, neurológicas, circulatórias, periféricas e múltiplas (ARTILHEIRO MMVSA, et al., 2014).

Segundo o Gráfico 3, a maioria dos pacientes possuem diagnóstico prévio de diabetes, assim, ao comparar os resultados com dados do Vigitel, 2016 (Vigilância de Fatores de Risco e Proteção para doenças crônicas por inquérito telefônico), nos quais $27,2 \%$ dos entrevistados com diagnóstico prévio de diabetes possuíam 65 anos ou mais, observa-se que em ambos estudos a faixa etária predominante é superior a 60 anos. Além disso, o envelhecimento da população, a crescente prevalência da obesidade e do sedentarismo e os processos de urbanização são considerados os principais fatores relacionados ao aumento da incidência e prevalência do diabetes mellitus tipo 2 em todo o mundo (COSTA AF, et al., 2017).

O conhecimento e a adesão ao regime terapêutico é um aspecto fundamental para um eficaz controle do Diabetes mellitus, em que o indivíduo tem um papel ativo e colaborativo no planejamento e implementação do regime de tratamento. Vários fatores interferem na adesão ao regime terapêutico (SOUSA MR, et al., 2015).

Acerca do tratamento do DM, a insulina é a base da terapia para indivíduos com diabetes tipo 1 e uma alternativa de tratamento para o diabetes tipo 2. Evidências recentes mostram que muitos pacientes com diabetes tipo 2 (DM2) inicialmente recusam o tratamento com insulina. Para alguns pacientes, isso pode levar a um controle glicêmico inadequado, enquanto outros podem reduzir a glicose no sangue por meios alternativos. (FLOREZ L, TURCHIN A, 2019).

Dentre os esquemas de insulinoterapia abordados nesse estudo, encontram-se a terapia de SlidingScale e o esquema Basal Bolus. A Terapia de SlidingScale trata a hiperglicemia após a ocorrência, com uma quantidade estabelecida de insulina administrada sem considerar o tempo do alimento, a presença ou ausência de administração pré-existente de insulina, ou mesmo a individualização da Sensibilidade do paciente à insulina, em vez de prevenir a ocorrência de hiperglicemia, uma abordagem que pode levar a mudanças rápidas nos níveis de glicemia, exacerbando hiperglicemia e hipoglicemia (CLEMENT S, et al., 2004).

Os pacientes tratados com insulina do esquema Basal Bolus (B-B), apresentaram maior melhora no controle glicêmico do que os tratados com SlidingScalelnsulin (SSI), em um estudo randomizado da terapia com insulina basal-bolus. As diferenças no controle glicêmico entre os grupos de tratamento podem ser explicadas pelo fato de que um regime de SSI trata a hiperglicemia após ter ocorrido em vez de prevenir a ocorrência de hiperglicemia (UMPIERREZ GE, et al., 2007).

Dessa forma, é notável que a literatura defenda o esquema Basal Bolus como o mais adequado, pois é eficaz no controle da hiperglicemia e leva a uma menor incidência de hipoglicemia. Porém, neste estudo podemos notar que a minoria dos pacientes (4\%) utilizou o esquema de insulina Basal Bolus isoladamente.

No presente estudo, os pacientes que utilizavam o esquema B-B obtiveram valores de glicemia inferiores aos que utilizavam o esquema SSI comprovando, então, a maior redução nos níveis de glicemia no tipo do esquema em questão. Os valores da média glicêmica encontrados foram inferiores aos encontrados por Umpierrez GE, et al (2007), sendo que, ambos estudos encontraram menor média glicêmica entre os pacientes que utilizaram o esquema B-B.

Esses resultados podem contribuir para o fato de que o esquema Basal-Bolus tenha maior eficácia terapêutica. É importante salientar que tais diferenças nas médias glicêmicas dos dois esquemas 
terapêuticos podem ser influenciadas pelo valor de glicemia dos pacientes, além disso, o número dos pacientes que fizeram uso do esquema basal bolus foi menor em relação aos pacientes que usaram o esquema SSI e esse fato pode ter contribuído para a diferença da média glicêmica assim como a ocorrência de hipoglicemias.

Existe uma relação direta entre dieta e controle glicêmico. Nesse estudo, todos os pacientes que utilizaram o esquema Basal-Bolus receberam uma dieta hipoglicêmica, fato que pode ter contribuído para ocorrência de uma menor média glicêmica nesse grupo. Além disso, apenas 26 pacientes (52\%) receberam dieta hipoglicêmica. Sabe-se que o conhecimento e controle sobre o tipo de dieta prescrita e seus horários são importantes para o planejamento terapêutico e prevenção da hiperglicemia (SOCIEDADE BRASILEIRA DE DIABETES, 2016).

Segundo revisão de Clement et al (2004), os objetivos do acompanhamento da dieta durante a hospitalização são otimizar o controle glicêmico, disponibilizar calorias necessárias para a demanda metabólica, além de auxiliar no acompanhamento nutricional após a alta. Quanto à frequência de hipoglicemia, esse estudo observou que a metade (50\%) dos pacientes que faziam uso do esquema B-B apresentaram, pelo menos, um episódio de hipoglicemia, enquanto, apenas $6,25 \%$ do grupo SSI apresentaram esse quadro. Mesmo com um número da amostra muito desigual entre os grupos, a análise estatística mostrou significância $(p=0,0400)$.

Episódios de hipoglicemia podem acontecer em doentes com ou sem diabetes que se encontram em ambiente hospitalar, e esse fator está ligado ao aumento de mortalidade (AKIROV A, et al., 2017). A hipoglicemia é comum em pacientes hospitalizados, sendo a insulina um importante fator de risco para sua ocorrência. Esses dados destacam a necessidade de pesar o risco de hipoglicemia contra o benefício de um controle glicêmico rígido ao considerar o início de insulina no hospital. (MILAN KP, et al., 2019).

A insulina basal utilizada neste estudo foi a NPH, com posologia de duas a três vezes ao dia. Essa insulina possui maior incidência de hipoglicemias em função do pico de ação em relação às outras dessa classe.A insulina Regular apresenta o inconveniente de início de ação mais retardado o que, no ambiente hospitalar, representa uma dificuldade a mais para a enfermagem, que deve aplicá-la 30 minutos antes da refeição, aumentando o risco de hipoglicemia, tanto por sua ação mais prolongada como pela imprevisibilidade do horário da dieta (GOMES PM, et al., 2014).

A hiperglicemia e hipoglicemia ocasionam riscos ao paciente internado, por isso é de grande importância o controle glicêmico através da insulinoterapia, para isso é preciso à escolha de um esquema eficaz. A equipe, por sua vez, deve estar capacitada para atender esses pacientes. Além disso, uma equipe multiprofissional deve auxiliar no cuidado ao paciente hospitalizado, buscando abordar diversas áreas visando maior atenção psíquica, maior controle da dor e sofrimento e cautela sobre medicações prescritas, uma vez que, algumas, podem ocasionar hiperglicemia.

Esses pontos podem levar a um maior controle glicêmico, o que é de grande importância, uma vez que, estudos recentes apontam que o controle da glicemia pode levar a um melhor resultado no prognóstico do paciente. (ZHAO L, et al., 2019).

\section{CONCLUSÃO}

Com este estudo foi possível detectar que a presença de hiperglicemia no ambiente intrahospitalar revelou-se mais prevalente em idosos. Além disso, nota-se que a grande maioria dos pacientes hospitalizados, que apresentam hiperglicemia, recebem insulina tipo SSI, um esquema não reconhecido pela literatura atual como o mais adequado. Portanto, o esquema ideal deveria garantir a correção e prevenção da hiperglicemia, além de evitar quadros de hipoglicemia. Foi encontrada uma diferença significativa na ocorrência de hipoglicemias, prevalecendo no grupo B-B. Porém, apesar disso, os grupos estudados eram muito desiguais quanto ao número de pacientes. Por fim, ressalta-se a importância de realizar novos estudos comparativos entre os esquemas insulinoterápicos, visando buscar aquele que se 
apresentasse como o mais eficaz. Além disso, seria interessante a elaboração de um algoritmo preciso e simples para a insulinoterapia hospitalar. Também é importante a realização de um trabalho de capacitação de toda a equipe envolvida e uma abordagem multidisciplinar visando, assim, a um cuidado de excelência a esses pacientes.

\section{REFERÊNCIAS}

1. AKIROV A, et al. Mortality among hospitalized patients with hypoglycemia: insulin related and noninsulin related. J Clin Endocrinol Metab. 2017; 102: 416-424.

2. ALFRADIQUE ME, et al. Internações por condições sensíveis à atenção primária: a construção da lista brasileira como ferramenta para medir o desempenho do sistema de saúde (Projeto ICSAP - Brasil). Cad. Saúde Pública, 2009; 25(6):1337-1349.

3. AMERICAN DIABETES ASSOCIATION. Pharmacologic Approaches to Glycemic Treatment: Standards of Medical Care in Diabetes-2018. Diabetes Care 2018; 41(1): S73-S85.

4. ARAÚJO LMB, et al. Tratamento do Diabetes Mellitus do Tipo 2: Novas Opções. Arquivo Brasileiro Endocrinologia e Metabologia, 2000; 44 (6): 509-518.

5. ARNOLD FW, et al. Hospitalization for community-acquired pneumonia: the pneumonia severity index vs clinical judgment, Chest. 2003; 124: 121- 124.

6. ARTILHEIRO MMVSA, et al. Quem são e como são tratados os pacientes que internam por diabetes mellitus no SUS? Saúde debate, 2014; 38(101): 210-224.

7. CLEMENT, S. et al. Management of diabetes and hyperglycemia in hospitals. Diabetes Care, 2004; $27(2): 553-591$.

8. COSTA AF, et al. Carga do diabetes mellitus tipo 2 no Brasil. Cad. Saúde Pública, 2017; 33 (2): e00197915.

9. FREITAS LRS, GARCIA LP. Evolução da prevalência do diabetes e deste associado à hipertensão arterial no Brasil: análise da Pesquisa Nacional por Amostra de Domicílios, 1998, 2003 e 2008. Epidemiologia e Serviços de Saúde,2012, 21(1): 7-19

10. FLOREZ L, TURCHIN A. Glycemic Control after Decline of Insulin Therapy by Patients. Diabetes, 2019; 68(1): 2376-PUB

11. GALINDO RJ, UMPIERREZ GE. Endocrine and metabolicmedical emergencies: a clinician's guide. Management of diabetes and/or hyperglycemia in non-critical care hospital settings. $2^{\text {nd }}$ es. New Jersey; Willey, 2018; $491-505$ p.

12. GELONEZE B, et al. Hiperglicemia pós-prandial: tratamento do seu potencial aterogênico. Arq. Bras. Cardiol. 2006; $87(5): 660-670$.

13. GÓES APP, et al. Diabetes mellitus tipo 1 no contexto familiar e social. Revista paulista de pediatria, 2007; 25(2):125-128.

14. GOMES PM, et al. Controle de hiperglicemia intra-hospitalar em pacientes críticos e não-críticos. Medicina (USP. FMRP), 2014; 47: 194-200.

15. KOVALASKE MA; GANDHI GY. Glycemic control in the medical intensive care unit. J Diabetes Sci Technol, $2009 ; 3$ (6):1330-1340.

16. LISBOA HRK, et al. Prevalência de hiperglicemia não diagnosticada nos pacientes internados nos hospitais de Passo Fundo, RS. Arq Bras Endocrinol Metab., 2000; 44(3): 220-226.

17. MACHADO SR, et al. Intervenção nutricional padronizada em pacientes hipoglicêmicos hospitalizados. Rev. Gaúcha Enferm., 2012; 33(1): 64-68.

18. MARRAS TK, et al. Applying a prediction rule to identify low-risk patients with community-acquired pneumonia. Chest, 2000; 118: 1339-1343.

19. MILAN KP, et al. Insulin Use and Hypoglycemia Prevalence in an Australian Public Hospital Setting. Diabetes Journal, 2019; 68: 2199-PUB.

20. PETERMANN XB, et al. Epidemiologia e cuidado à Diabetes Mellitus praticado na Atenção Primária à Saúde: uma revisão narrativa. Saúde [periódico na Internet], 2015; 41(1): 49-56.

21. ROSA R, et al. Estimated hospitalizations attributable to Diabetes Mellitus within the public healthcare system in Brazil from 2008 to 2010: study DIAPS 79. Rev. Assoc. Med. Bras., 2014; 60(3): 222-230.

22. Sociedade Brasileira de Diabetes. Diretrizes da Sociedade Brasileira de Diabetes (2015-2016). São Paulo: A.C. Farmacêutica, 2016.

23. SOUSA MR, et al. Questionário dos conhecimentos da Diabetes (QCD): propriedades psicométricas. Revista Portuguesa de Saúde Pública, 2015; 33: 33-41.

24. UMPIERREZ GE, et al. Randomized study of basal-bolus insulin therapy in the inpatient management of patients with type 2 diabetes. Diabetes Care, 2007, 30: 2181-2186.

25. VIANA MV, et al. Avaliação e tratamento da hiperglicemia em pacientes graves. Rev. bras. ter. intensiva, 2014; 26(1): 71-76.

26. ZHAO L, et al. Hyperglycemia is associated with poor in-hospital outcome in elderly patients with acute ischemic stroke. Medicine, 2019; 98(31): e16723. 